\title{
A meta-analysis of robot assisted laparoscopic radical prostatectomy versus laparoscopic radical prostatectomy
}

https://doi.org/10.1515/med-2019-0052

received August 25, 2018; accepted October 13, 2018

\begin{abstract}
Objective: To evaluate the efficacy and safety of robot-assisted laparoscopic radical prostatectomy (RALP) and laparoscopic radical prostatectomy (LRP) for the treatment of localized prostate cancer. Methods: Meta-analysis was applied using Review Manager V5.3 software and the retrieved clinical trials comparing RALP with LRP for the treatment of localized prostate cancer published from 2000 to 2018 in PubMed, Ovid, ScienceDirect, and EMBASE datasets were analyzed. Results: This meta-analysis included 16 articles, totaling 7952 patients, with 5170 RALP patients and 2782 LRP patients. Meta-analysis showed that RALP postoperative complications were fewer $(\mathrm{P}=0.0007)$, and the postoperative urinary continence rate was better at 1 year after surgery $(\mathrm{P}<0.00001)$. There was no statistical significance between RALP and LRP with regards to the positive incidence of surgical margin $(\mathrm{P}=$ 0.18). Conclusion: As an emerging technology, RALP is superior to LRP for localized prostate cancer treatment in terms of postoperative complications, and postoperative urinary continence rate.
\end{abstract}

Keywords: Prostate cancer; Robot-assisted laparoscopic; Laparoscopy; Meta-analysis

\section{Introduction}

Prostate cancer is one of the most common malignant tumors in older men [1]. At present, the main surgical

\footnotetext{
*Corresponding author: Songtao Wang, Armed police Beijing Corps Hospital, Beijing 100027, China, Email: songtaowwj@126. com

Tao Wang, Qunsuo Wang, First Department of Surgery, Armed police Beijing Corps Hospital, Beijing 100027, China
}

treatment methods include open surgery, retro-pubic laparoscopic radical prostatectomy, and robotic-assisted laparoscopic radical prostatectomy [2]. With the rapid development of minimally invasive techniques, laparoscopic techniques have been increasingly applied to urological surgery [3], and the most important minimally invasive procedures include retropubic laparoscopic radical prostatectomy (LRP) and robotic assisted laparoscopic prostate (RALP), both of which have the advantages of small surgical incisions, less trauma, less bleeding, fewer postoperative complications, faster recovery, shorter hospital stay, etc.. However, which technique is better is still controversial. For this reason, this study used the meta analysis method to provide an evidence-based medical basis for treatment decisions regarding clinical prostate cancer.

\section{Methods}

\subsection{Searching method}

We conducted a search of PubMed, Ovid, ScineceDirect, and EMBASE from 2005 to 2018. We developed a search strategy as outlined by the Cochrane Collaboration Workbook. The medical subject heading terms and keywords included "prostate cancer prostatectomy", "radical prostatectomy laparoscopy”, “assisted-laparoscopic”. We excluded duplicate articles and unpublished studies from international conferences.

\subsection{Inclusion criteria}

(1) The patients that were diagnosed with localized prostate cancer treated for the first time, and all had indications for treatment by radical prostatectomy. (2) The literature compared the difference in efficacy between RALP and LRP. (3) The study was designed as a prospective controlled study or a retrospective case-control study. (4) 
The literature involved at least one of the following seven indicators: operation time, intraoperative blood loss, blood transfusion rate, surgical margin positive rate and hospitalization time, postoperative urinary function and postoperative complications, and follow-up time more than 1 year.

\subsection{Exclusion criteria}

The following criteria were used to exclude literature: non-clinical controlled trials, non-prostate cancer studies, other surgical treatments or data descriptions were unclear.

\subsection{Data extraction}

The literature was selected according to the inclusion/ exclusion criteria, and the parallel extraction method was used, which was completed by two researchers and cross-examined. When the opinions were inconsistent, decisions to include or exclude literature were resolved through discussion. Any lack of information was supplemented by contact with the author, which included basic information about the subject, including patient numbers, characteristics, interventions, and outcome measures. Literature quality evaluation: The methodological quality of the randomized controlled trial literature included in the study was based on the Jadad quality scoring method, including randomization, blinding, etc.

\subsection{Statistical analysis}

Meta analysis was performed using RevMan 5.3 software recommended by the Cochrane Collaboration. If the two categorical variables were used, the odds ratios and their 95\% confidence intervals were used to describe the results. $\mathrm{P}<0.05$ was considered statistically significant. If it was a continuous variable, the weighted mean difference (WMD) was used to describe the results. The included studies were simultaneously tested for homogeneity. If the study data was not heterogeneous, a fixed effect model is used; if heterogeneity exists, the sensitivity analysis is used to filter heterogeneous study heterogeneity. If there was clinical consistency, a random effect model was used, and vice versa, descriptive analysis is used.

\section{Results}

\subsection{Literature searches and characteristics of eligible studies}

Figure 1 shows the flow diagram of the study selection process. After further screening, we obtained 22 studies. Table 1 summarizes the detailed characteristics of the 22 eligible studies.

Table 1: Baseline characteristics of included studies

\begin{tabular}{|c|c|c|c|}
\hline References & Group & NO. of patients & Mean age (years) \\
\hline \multirow[t]{2}{*}{ Coelho 2010 [9] } & RALP & 250 & 60.4 \\
\hline & LRP & 250 & 62.9 \\
\hline \multirow[t]{2}{*}{ Drouin 2009 [10] } & RALP & 71 & 60.4 \\
\hline & LRP & 85 & 61.8 \\
\hline \multirow[t]{2}{*}{ Ficarra 2007 [11] } & RALP & 40 & 62.8 \\
\hline & LRP & 40 & 63.1 \\
\hline \multirow[t]{2}{*}{ Finkelstein 2010 [12] } & RALP & 2841 & 61.3 \\
\hline & LRP & 168 & 63.6 \\
\hline \multirow[t]{2}{*}{ Hakimi 2009 [13] } & RALP & 75 & 59.8 \\
\hline & LRP & 75 & 59.6 \\
\hline \multirow[t]{2}{*}{ Hu 2006 [14] } & RALP & 322 & 63.7 \\
\hline & LRP & 358 & 62.1 \\
\hline \multirow[t]{2}{*}{ Joseph 2005 [15] } & RALP & 50 & 59.6 \\
\hline & LRP & 50 & 61.8 \\
\hline \multirow[t]{2}{*}{ Magheli 2011 [16] } & RALP & 522 & 58.3 \\
\hline & LRP & 522 & 58.4 \\
\hline \multirow[t]{2}{*}{ Menon 2002 [17] } & RALP & 40 & 60.7 \\
\hline & LRP & 40 & 62.8 \\
\hline \multirow[t]{2}{*}{ Papachristos 2015 [18] } & RALP & 100 & 60.5 \\
\hline & LRP & 100 & 62.5 \\
\hline \multirow[t]{2}{*}{ Park 2013 [19] } & RALP & 183 & 63 \\
\hline & LRP & 144 & 67 \\
\hline \multirow[t]{2}{*}{ Porpiglia 2013 [20] } & RALP & 60 & 63.9 \\
\hline & LRP & 60 & 64.7 \\
\hline \multirow[t]{2}{*}{ Rozet 2007 [21] } & RALP & 133 & 62 \\
\hline & LRP & 133 & 62.5 \\
\hline \multirow[t]{2}{*}{ Tozawa 2014 [22] } & RALP & 157 & 67 \\
\hline & LRP & 551 & 67.4 \\
\hline \multirow[t]{2}{*}{ Trabulsi 2011 [23] } & RALP & 205 & 59.9 \\
\hline & LRP & 45 & 58.1 \\
\hline \multirow[t]{2}{*}{ Willis 2012 [24] } & RALP & 121 & 58.1 \\
\hline & LRP & 161 & 58 \\
\hline
\end{tabular}




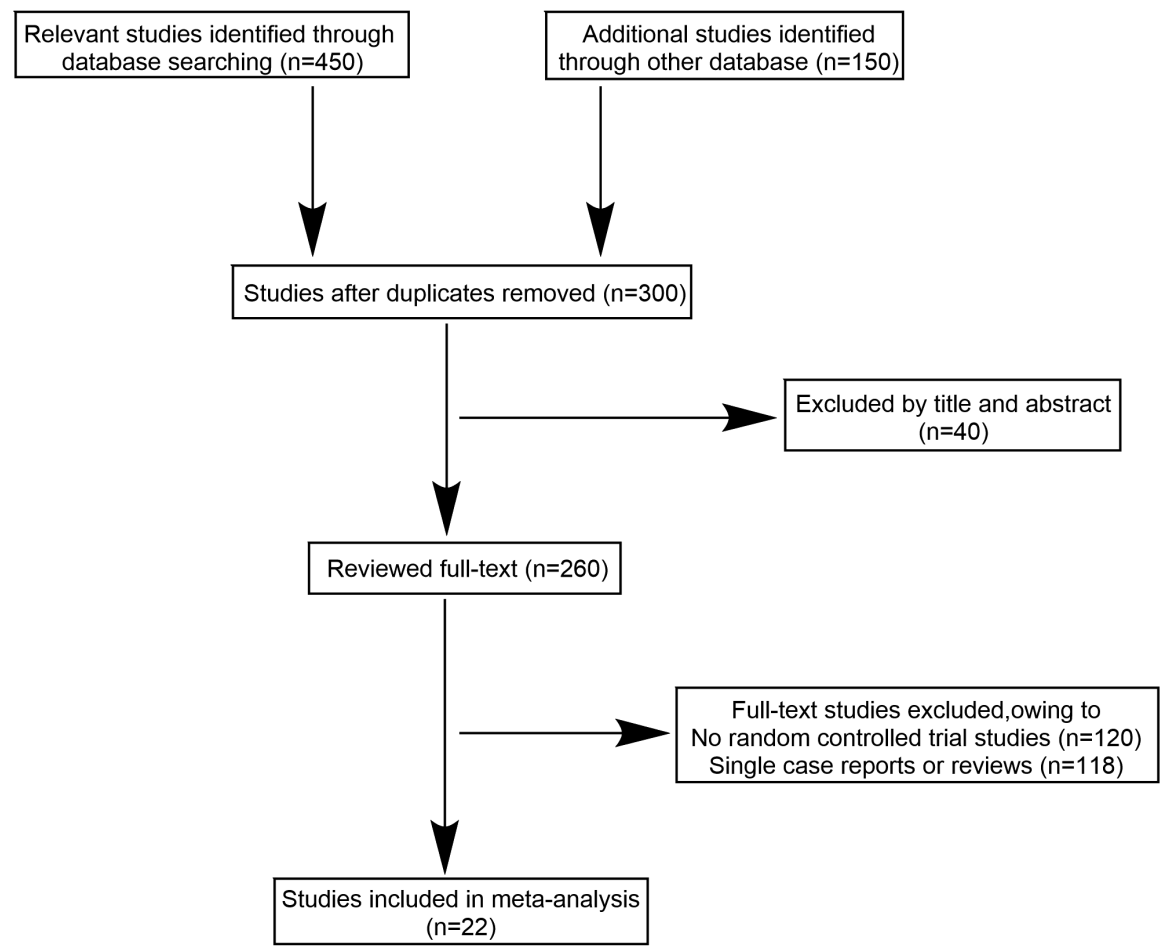

Figure 1: The study selection process

\subsection{Meta-analysis of surgical blood loss of RALP and LRP}

Surgical blood loss was reported in nine studies (Figure 2). Compared with LRP, RALP was associated with a 0.38 reduction in blood loss (95\% CI, [-0.84, 0.08]. Significant heterogeneity was found among these studies $\left(\mathrm{I}^{2}=95 \%, \mathrm{P}\right.$ $<0.00001)$. Due to significant heterogeneity of the data, we used a random effect model. According to our analysis, the difference between RALP and LRP was not significant.

\subsection{Meta-analysis of the postoperative com- plications of RALP and LRP}

The number of postoperative complications was reported in eight studies (Figure 3). Data from these 8 studies was analyzed in a fixed effect model and the pooled OR was 0.57 (95\% CI, [0.46, 0.70]). The $\mathrm{I}^{2}$ estimate of the variance between the studies is $35 \%$ and $\mathrm{P}=0.16$, which showed low heterogeneity. According to our analysis, the postoperative complications between RALP and LRP was significant. $(\mathrm{P}<0.00001)$.

\subsection{Meta-analysis of the postoperative urinary continence rate 1 year after RALP and LRP}

The postoperative urinary continence rate 1 year after surgery was reported in eight studies (Figure 4). The fixed effects model was used. The meta-analysis demonstrated that patients with RALP had significantly lower postoperative urinary continence rates 1 year after surgery compared with LRP $(\mathrm{OR}=2.09$; 95\% CI, $[1.61,2.73])$. The $\mathrm{I}^{2}$ estimate of the variance between these studies is $0 \%$ and $\mathrm{P}=$ 0.73, which showed no significant heterogeneity. According to our analysis, the postoperative urinary continence rate 1 year after RALP was lower than LRP $(\mathrm{P}<0.00001)$.

\subsection{Meta-analysis of the rate of positive surgical margins of RALP and LRP}

The rate of positive surgical margins was reported in twelve studies (Figure 5). The fixed effects model was used. The meta-analysis shows that the rate of positive surgical margins of RALP and LRP was not significant (P $=0.82)$ and the pooled OR was 0.97 (95\% CI, [0.76, 1.24]). The $\mathrm{I}^{2}$ is $43 \%$ and $\mathrm{P}=0.06$, which indicate low heterogeneity. According to our analysis, the rate of positive surgical margins of RALP and LRP was not significant $(\mathrm{P}=0.82)$. 




Figure 2: The forest plot for surgical blood loss of RALP and LRP groups

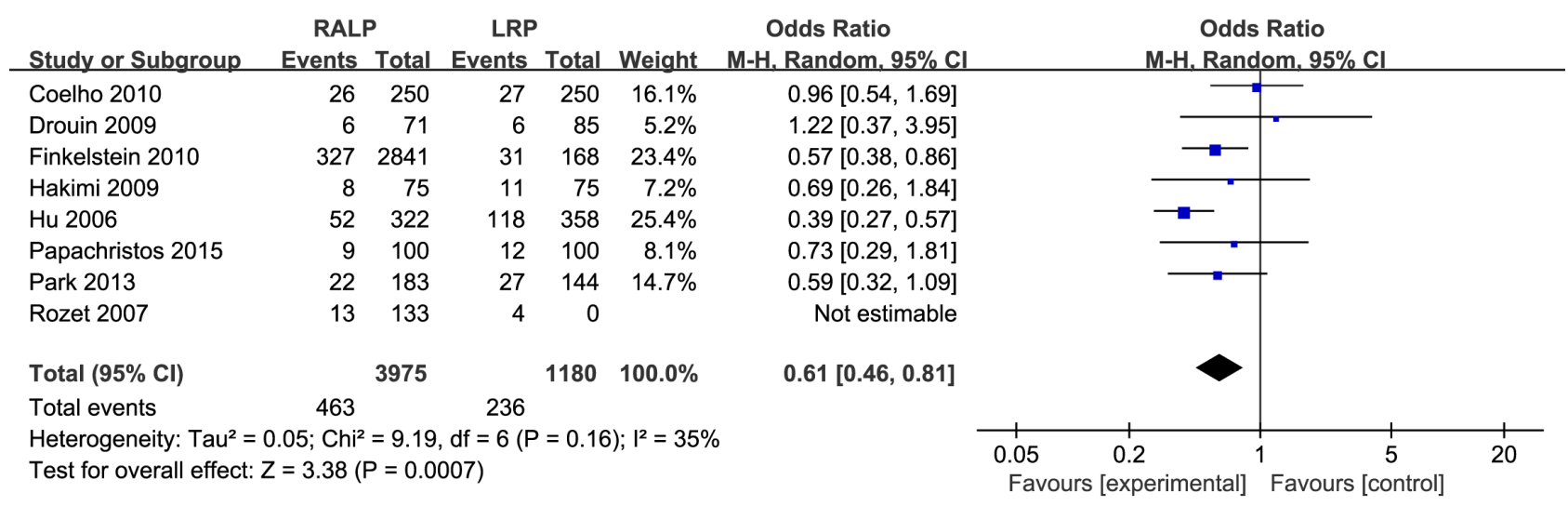

Figure 3: The forest plot for postoperative complications of RALP and LRP groups

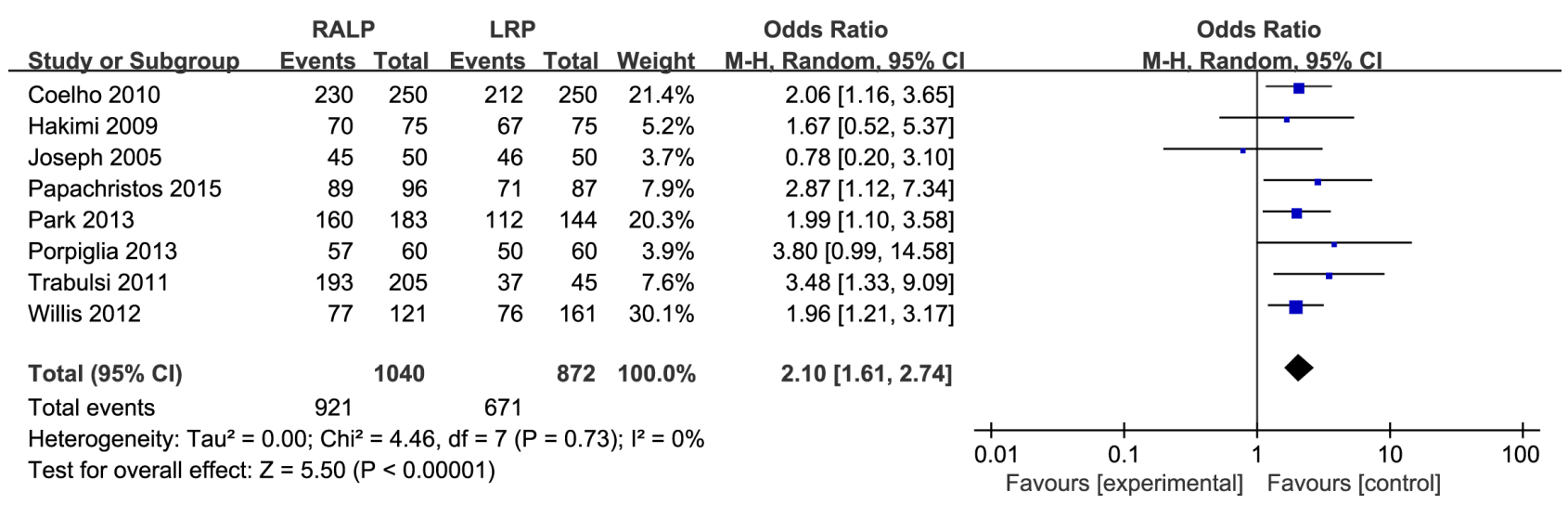

Figure 4: The forest plot for the postoperative urinary continence rate 1 year after RALP and LRP

\section{Discussion}

Because patients and their family members have the right to self-selection (taking into account economic and local medical conditions, etc.) [4], it is unrealistic to find completely randomized controlled studies. Therefore, most of the literature we have included are prospective and non-random controlled trials or retrospective controlled studies.

There are many complications after radical prostatectomy, including anastomotic leakage, anastomotic stenosis, rectal injury, urinary incontinence, and erectile dysfunction [5]. Due to the limited number of published studies and data values, this study analyzed the incidence 


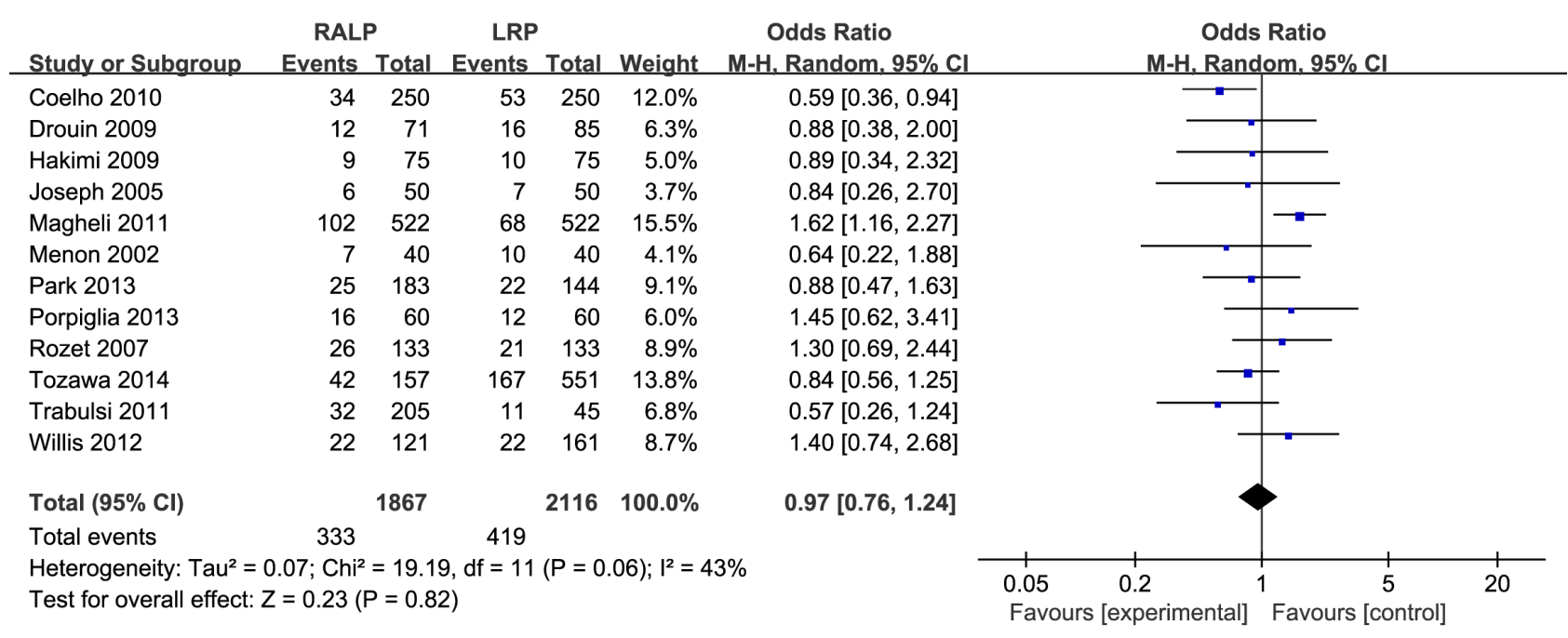

Figure 5: The forest plot for the rate of positive surgical margins of RALP and LRP

of postoperative complications in general, and the results showed that robot-assisted laparoscopy was lower.

The main advantage of robot-assisted laparoscopic surgery is to simulate the standard steps of traditional laparoscopic surgery [6]. In the narrow pelvic space, the flexible robotic arm makes the anatomical operation finer than the human hand, and it is easier to preserve the integrity of the nerve [7]. The urinary structure is maintained in tumor resection and sexual function and complete urinary control is retained. However, its main disadvantages are high cost (including cost, maintenance and consumables). The cost problem has limited the widespread promotion of robot-assisted laparoscopy in the clinic [8], but we believe that it may be reduced in the future through improvement.

Limitations of this paper and future research directions: Due to the limitation of the number and quality of the included literature, and the lack of grey literature, these factors may cause deviation of the analysis results; therefore, it is necessary to further study the long-term follow-up effects of the two procedures. This should provide more strong evidence for clinical practice and research work.

In summary, robot-assisted laparoscopic surgery is safer and more effective than laparoscopic radical prostatectomy, but more prospective randomized controlled trials are needed for further validation. Looking to the future, robotic assistive technology will be more widely used in the clinical treatment of prostate cancer.

Conflict of interest: Authors state no conflict of interest.

\section{References}

[1] Garg T, Young AJ, Kost KA, Danella JF, Larson S, Nielsen ME, et al. Burden of multiple chronic conditions among patients with urological cancer. The Journal of urology 2018;199(2):543-550

[2] Porcaro AB, Molinari A, Terrin A, De Luyk N, Baldassarre R, Brunelli $M$, et al. Robotic-assisted radical prostatectomy is less stressful than the open approach: results of a contemporary prospective study evaluating pathophysiology of cortisol stress-related kinetics in prostate cancer surgery. Journal of robotic surgery 2015;9(3):249-255

[3] Wright GP, Zureikat AH. Development of Minimally Invasive Pancreatic Surgery: an Evidence-Based Systematic Review of Laparoscopic Versus Robotic Approaches. Journal of Gastrointestinal Surgery 2016;20(9):1-8

[4] Ms AK, Md TA, Phd YM, Md HK. Family functioning perceived by patients and their family members in three Diagnostic and Statistical Manual-IV diagnostic groups. Psychiatry \& Clinical Neurosciences 2010;58(5):495-500

[5] Shelbaia A, Salem HK, Emran A, Raouf MA, Rahman SA. Long term complications after radical cystoprostatectomy with orthotopic diversion in male patients: Preliminary experience. African Journal of Urology 2013;19(2):89-93

[6] Grasso AAC, Lorenzis ED, Randi G, Rosso M, Cozzi G, Zaramella S, et al. 524 Assessment of predictive factors of perioperative complications after minimally invasive radical prostatectomy: A multicentre study. European Urology Supplements 2014;13(1):e524-e524

[7] Mudrov VA, Chatskis EM, Nizhegorodtseva DA, Tttjan EV. Significance of ultrasound pelviometry in the diagnostics of anatomical and clinical narrow pelvis. 2017;66(6):20-29

[8] Park JW, Choi KH, Yang SC, Han WK. Cost Aspects of Radical Nephrectomy for the Treatment of Renal Cell Carcinoma in Korea: Open, Laparoscopic, Robot-Assisted Laparoscopic, and Video-Assisted Minilaparotomy Surgeries. Korean Journal of Urology 2012;53(8):519-523

[9] Coelho RF, Rocco B, Patel MB, Orvieto MA, Chauhan S, Ficarra V, et al. Retropubic, laparoscopic, and robot-assisted 
radical prostatectomy: a critical review of outcomes reported by high-volume centers. Journal of Endourology 2010;24(12):2003-2015

[10] Drouin SJ, Vaessen C, Hupertan V, Comperat E, Misraï V, Haertig A, et al. Comparison of mid-term carcinologic control obtained after open, laparoscopic, and robot-assisted radical prostatectomy for localized prostate cancer. World journal of urology 2009;27(5):599-605

[11] Ficarra V, Cavalleri S, Novara G, Aragona M, Artibani W. Evidence from robot-assisted laparoscopic radical prostatectomy: a systematic review. European urology 2007;51(1):45-56

[12] Finkelstein J, Eckersberger E, Sadri H, Taneja SS, Lepor H, Djavan B. Open versus laparoscopic versus robot-assisted laparoscopic prostatectomy: the European and US experience. Reviews in urology 2010;12(1):35

[13] Hakimi AA, Blitstein J, Feder M, Shapiro E, Ghavamian R. Direct comparison of surgical and functional outcomes of robotic-assisted versus pure laparoscopic radical prostatectomy: single-surgeon experience. Urology 2009;73(1):119-123

[14] Hu JC, Nelson RA, Wilson TG, Kawachi MH, Ramin SA, Lau C, et al. Perioperative complications of laparoscopic and robotic assisted laparoscopic radical prostatectomy. The Journal of urology 2006;175(2):541-546

[15] Joseph JV, Vicente I, Madeb R, Erturk E, Patel HR. Robotassisted vs pure laparoscopic radical prostatectomy: Are there any differences? BJU international 2005;96(1):39-42

[16] Magheli A, Gonzalgo ML, Su LM, Guzzo TJ, Netto G, Humphreys EB, et al. Impact of surgical technique (open vs laparoscopic vs robotic-assisted) on pathological and biochemical outcomes following radical prostatectomy: an analysis using propensity score matching. BJU international 2011;107(12):1956-1962
[17] Menon M, Shrivastava A, Tewari A, Sarle R, Hemal A, Peabody JO, et al. Laparoscopic and robot assisted radical prostatectomy: establishment of a structured program and preliminary analysis of outcomes. The Journal of urology 2002;168(3):945-949

[18] Papachristos A, Basto M, te Marvelde L, Moon D. Laparoscopic versus robotic-assisted radical prostatectomy: an A ustralian single-surgeon series. ANZ journal of surgery 2015;85(3):154-158

[19] Park B, Kim W, Jeong BC, Jeon SS, Lee HM, Choi HY, et al. Comparison of oncological and functional outcomes of pure versus robotic-assisted laparoscopic radical prostatectomy performed by a single surgeon. Scandinavian journal of urology 2013;47(1):10-18

[20] Porpiglia F, Morra I, Chiarissi ML, Manfredi M, Mele F, Grande $\mathrm{S}$, et al. Randomised controlled trial comparing laparoscopic and robot-assisted radical prostatectomy. European urology 2013;63(4):606-614

[21] Rozet F, Jaffe J, Braud G, Harmon J, Cathelineau X, Barret E, et al. A direct comparison of robotic assisted versus pure laparoscopic radical prostatectomy: a single institution experience. The Journal of urology 2007;178(2):478-482

[22] Tozawa K, Yasui T, Umemoto Y, Mizuno K, Okada A, Kawai $\mathrm{N}$, et al. Pitfalls of robot-assisted radical prostatectomy: A comparison of positive surgical margins between robotic and laparoscopic surgery. International Journal of Urology 2014;21(10):976-979

[23] Trabulsi E, Zola J, Colon-Herdman A, Heckman J, Gomella L, Lallas C. Minimally invasive radical prostatectomy: transition from pure laparoscopic to robotic-assisted radical prostatectomy. Archivos espanoles de urologia 2011;64(8):823-829

[24] Willis DL, Gonzalgo ML, Brotzman M, Feng Z, Trock B, Su LM. Comparison of outcomes between pure laparoscopic vs robot-assisted laparoscopic radical prostatectomy: A study of comparative effectiveness based upon validated quality of life outcomes. BJU international 2012;109(6):898-905 